Article

\title{
Anticancer activity and structure activity relationship of non-symmetrical choline kinase inhibitors.
}

\author{
Santiago Schiaffino-Ortega ${ }^{1+}$, Elena Mariotto ${ }^{3+}$, Pilar María Luque-Navarroo ${ }^{1+}$, María Kimatrai \\ Salvador1, Ramon Hurtado-Guerrero", Pablo Rios-Marco, Carmen Marco, María Paz \\ Carrasco-Jimenez ${ }^{2 *}$, Giampietro Viola ${ }^{3 *}$, Luisa Carlota López-Cara ${ }^{*}$ \\ 1 Department of Pharmaceutical and Organic Chemistry, Faculty of Pharmacy, Campus of Cartuja, 18071

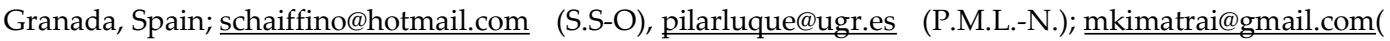 \\ M.K-S) \\ 2 Department of Biochemistry and Molecular Biology I, Faculty of Sciences, 18071, Granada, Spain \\ priosm@ugr.es (P.R.M), cmarco@ugr.es (C.M) \\ 3 Department of Woman's and Child's Health, Laboratory of Oncohematology, University of Padova, 35128, \\ Padova, Italy elena.mariotto@unipd.it (E.M) \\ 4 Institute of Biocomputation and Physics of Complex Systems (BIFI), University of Zaragoza, BIFI-IQFR \\ (CSIC) Joint Unit, Mariano Esquillor s/n, Campus Rio Ebro, Edificio I+D; Fundacion ARAID, Edificio \\ Pignatelli 36 (Spain). rhurtado@bifi.es (R. H-G) \\ * Correspondence: mpazcj@ugr.es (M.P.C.-J.); giampietro.viola.1@unipd.it (G.V); lcarlotalopez@ugr.es \\ (L.C.L.-C.)Tel.: +34-958-243-248 (M.P.C.-J.); +34-958-243-849(L.C.L.-C.) \\ + These authors contributed equally to this work
}

\begin{abstract}
Choline kinase inhibitors are an important class of cytotoxic compounds useful for the treatment of different forms of cancer since aberrant choline metabolism is a feature of neoplastic cells. Here we present the characterization and the structure activity relationship of a series of non-symmetrical choline kinase inhibitors characterized by a 3-aminophenol moiety, bound to 4-(dimethylamino)- or 4-(pyrrolidin-1-yl)pyridinium cationic heads through several linkers. These derivatives were evaluated both for their inhibitory activity on the enzyme and for their antiproliferative activity in a panel of six human tumor cell lines. The compounds with the best inhibitory results were those connected to the linker by the $\mathrm{N}$-atom (4a-h) and these results are supported by docking studies. The compounds with the best antiproliferative results were those connected to the linker by the O-atom (3a-h). On the other hand, as was predictable in both families, the inhibitory effect on the enzyme is greater the shorter the length of the linker, while in tumor cells, lipophilicity and choline uptake inhibition could play a decisive role.
\end{abstract}

Interestingly compounds $\mathbf{3 c}$ and $\mathbf{4 f}$, selected for both their ability to inhibit the enzyme and good antiproliferative activity, are endowed with a low toxicity in non-tumoral cells (e.g human peripheral lymphocytes) respect to cancer cells. These compounds were also able to induce to induce apoptosis in Jurkat leukemic cells without causing significative variations of cell cycle. It is worth to mention that these derivatives, beside their inhibitory effect on choline kinase, displayed a modest ability to inhibit choline uptake thus suggesting that this mechanism may also contribute to the observed cytotoxicity.

Keywords: Antitumoral drug, Choline kinase inhibition, Choline uptake

\section{Introduction}

Membranes of eukaryotic cells are composed principally by phospholipids and the zwitterionic glycerophospholipids phosphatidylcholine (PC) and phosphatidylethanolamine (PE) are the two 
most common phospholipids species in this type of cells. PC, the most abundant phospholipid class of cell membrane, constitutes likewise the major phospholipid class contained in lipoproteins, biliary lipids aggregates and lung surfactant. [1,2] PC serves as a direct substrate for sphingomyelin (SM) synthesis and it is also a fundamental source of the second messenger diacylglycerol (DAG), phosphatidic acid, lysophosphatidic acid and arachidonic acid which can be further metabolized to other signaling molecules. PC has also been reported to be a mitogen required for DNA synthesis induced by growth factors [1]. PC can be synthesized via the de-novo pathway, also known as the Kennedy pathway and responsible also of the synthesis of PE from ethanolamine, from the quaternary amine choline (see Scheme 1). The 95\% of the total pool of choline located in most tissues will enter in this metabolic route [1] whose first step is catalyzed by the choline kinase (ChoK) enzyme. ChoK is a cytosolic enzyme that catalyzes the ATP-dependent phosphorylation of choline in the presence of $\mathrm{Mg}^{++}[1,3]$.

The choline kinase family in humans comprises two chok genes: chok- $\alpha$ and chok- $\beta$ genes which encode three isoforms; ChoK $\alpha 1$ (457aa, 52kDa), Chok $\alpha 2$ (439aa, 50kDa) and ChoK $\beta$ (395aa, 45kDa). While ChoK $\alpha 1$ and $\mathrm{ChoK} \alpha 2$ derived from the same gene by differential splicing and are almost identical (except that ChoK $\alpha 1$ has an extra of 18 amino acids), the homology between ChoK $\alpha$ and ChoK $\beta$ is $60 \%$, but it is higher in the choline domain $[3,4]$.

Normal cell proliferation is characterized by the strict duplication of all cellular components in a strict temporal order. In the particular case of lipids, the maintenance of the status of PC as one of the structural elements of cell membranes and source of important mitotic second messengers would have important implications in cell proliferation and apoptosis [1,5]. Actually, among other changes, tumors display elevated levels of phospholipids characterized by the increase of phosphocholine (PCho) and total choline containing metabolites [6-8] ChoK $\alpha$ appears overexpressed in 40-60\% of breast cancer, prostate, lung, colon, ovarian and bladder which represent $70 \%$ of all cancers in developed countries. For this reason, ChoK has been proposed a prognostic marker progression [3] as well as a molecular target in oncology [9-13]. 

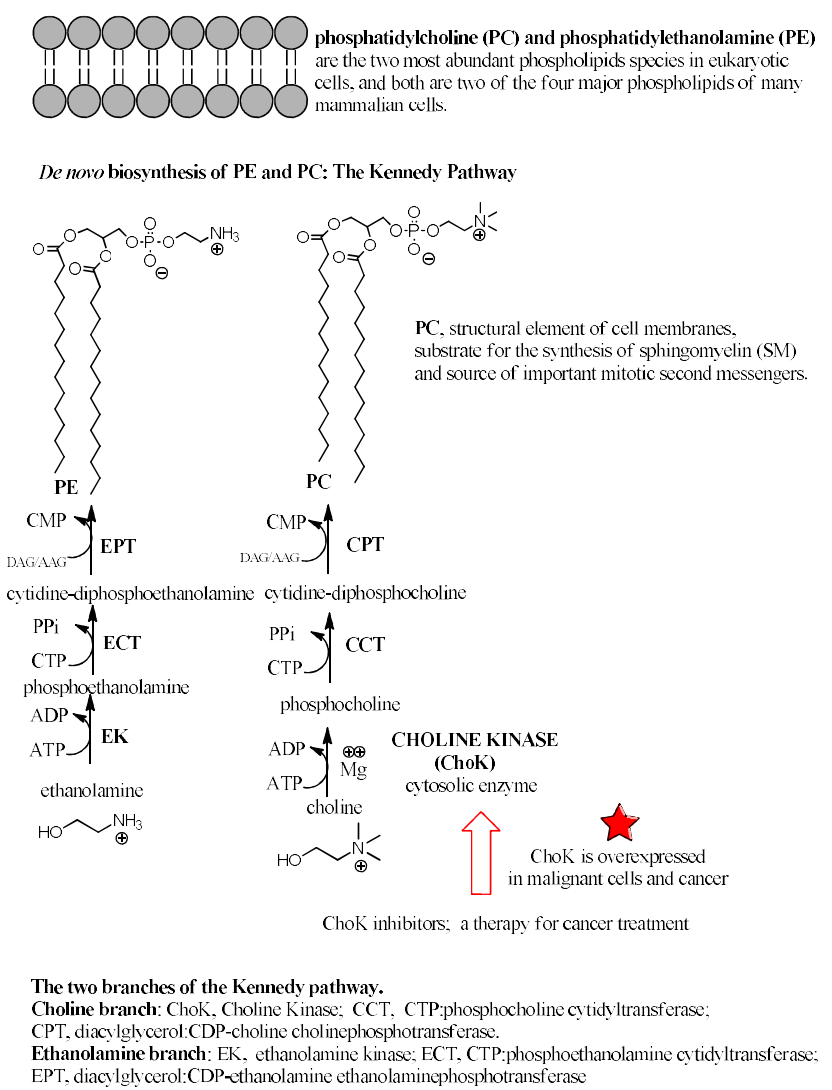

Scheme 1. The Kennedy Pathway or the de-novo biosynthesis of phosphatidylcholine (PC) and phosphatidylethanolamine (PE).

Due to its vital and widely studied role in cell division as well in tumor formation, ChoK emerged as potential target for various cancers [7-9] particularly Ras-induced carcinogenesis. The Ras effectors serine/threonine kinase (Raf-1), the Ral-GDP dissociation stimulator (Ral-GDS) and the phosphatidylinositol 3-kinase (PI3K) all are involved in ChoK activation during tumorigenesis [3, $5,14]$.

We have previously reported the synthesis and the biological evaluation of a new family structurally related to hemicolinium-3 (HC-3) of non-symmetrical monocationic compounds (Figure 1) endowed with antitumor activity including a 3-amino-phenol moiety bound to 4-pyrrolidinopyridinium or 4-dimethylaminopyridinium groups through several linkers [15]. In the last decade there have been numerous studies on ChoK $\alpha 1$ inhibitors published and their great applicability in different diseases. For this reason, we have been encouraged to further develop our compound library. 


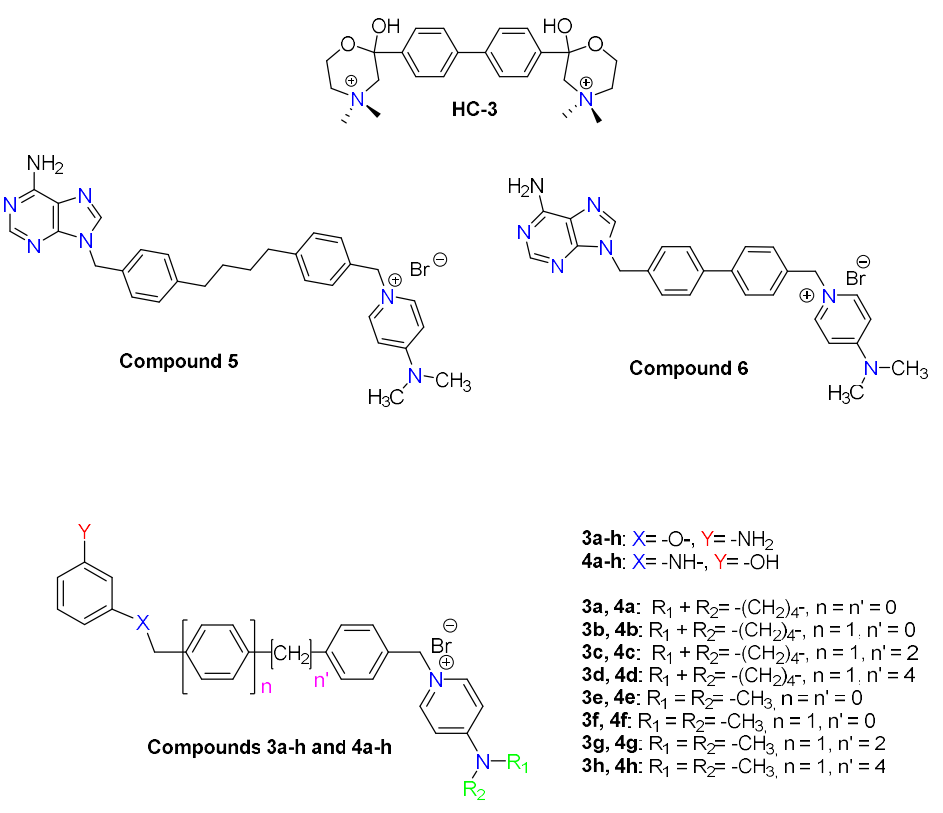

Figure 1. Structures of hemicolinium-3 (HC-3), the monocationic compounds 5 and $\mathbf{6}$ and monocationic $3 \mathbf{a}-\mathbf{h}$ and $\mathbf{4 a - h}$ structures. [15].

The residues that constitute the ATP binding site in the enzyme is quite different to those residues that form the Cho binding site, thus suggesting the idea of synthesizing non-symmetric monocationic inhibitors which should have one cationic head that could be inserted into the Cho binding site and another fragment that could mimic the ATP adenine moiety, having into account that the size of symmetrical biscationic inhibitors was appropriated to bind simultaneously in both the ATP and choline putative binding sites of the protein model [15-20]. On the other hand, we have recently reported a new series of small monocationic molecules where the inhibition of choline uptake has emerged as a major contributor to the antiproliferative outcome of this class of compounds [9]. The results provided in the present study complement the outcomes earlier reported since docking studies have been done in more appropriate crystal structures, the inhibitory activity have been described over the isolate ChoK $\alpha 1$ enzyme and the antiproliferative effects of derivatives have been tested against a panel of six human cancer cell lines in order to complete and validate the earlier experiments reported.

\section{Materials and Methods}

\subsection{Chemistry}

The compounds $\mathbf{3 a}-\mathbf{h}$ and $\mathbf{4 a}-\mathbf{h}$ were synthesized following the protocols described previously [15].

\subsection{Cloning, purification of $C K$ and Inhibition of Choline Kinase $\alpha 1$ by compounds $\mathbf{3} \boldsymbol{a}-\boldsymbol{h}$ and $\mathbf{4} \boldsymbol{a} \boldsymbol{-} \boldsymbol{h}$.}

Details about cloning and purification of human $\mathrm{ChoK} \alpha 1$ and $\mathrm{ChoK} \beta$ have been previously reported [17].

The effect of compounds $\mathbf{3 a}-\mathbf{h}$ and $\mathbf{4 a} \mathbf{a}-\mathbf{h}$ on ChoK $\alpha 1$ was assayed in purified ChoK $\alpha 1$ as previously described $[17,19,20]$, by determining the rate of incorporation of ${ }^{14} \mathrm{C}$ from [methyl $1{ }^{14} \mathrm{C}$ ]choline into phosphocholine, both in the absence (control) or presence of different inhibitor concentrations. 
Briefly, $20 \mathrm{ng}$ of purified ChoK $\alpha 1$ were incubated with $1 \mathrm{mM}$ [methyl- $\left.{ }^{14} \mathrm{C}\right]$ choline $(4500 \mathrm{dpm} / \mathrm{nmol})$ in $100 \mathrm{mM}$ Tris- $\mathrm{HCl}$ ( $\mathrm{pH} \mathrm{8.5),} 10 \mathrm{mM} \mathrm{MgCl}$, $10 \mathrm{mM} \mathrm{ATP}$, and incubated at $37^{\circ} \mathrm{C}$ for $10 \mathrm{~min}$. The reaction was stopped by immersing the reaction tubes in boiling water for $3 \mathrm{~min}$. Aliquots of the reaction were applied to the origin of silica gel plates in the presence of phosphocholine $(0.1 \mathrm{mg})$ and choline $(0.1 \mathrm{mg})$ as carriers. The chromatography was developed in methanol/0.6\% $\mathrm{NaCl} / 28 \%$ $\mathrm{NH}_{4} \mathrm{OH}$ in water (50:50:5, v/v/v) as solvent, and phosphocholine was visualized under exposure to iodine vapor. The corresponding spot was scraped and transferred to scintillation vials for measurement of radioactivity by a Beckman 6000-TA (Madrid, Spain) liquid-scintillation counter. The $50 \%$ inhibitory concentrations ( $\mathrm{IC}_{50}$ value) were determined from the \% activity of the enzyme at different concentrations of synthetic inhibitor by using a sigmoidal dose-response curve (the ED50plus v1.0 software).

\subsection{Docking studies}

The computationl studies have been performed as previuly reported [17-19].

\subsection{Antiproliferative assays in cancer cells}

Human cervix carcinoma (HeLa) and human breast cancer (MCF-7) were grown in DMEM medium (Gibco, Milan, Italy). B-acute lymphoblastic leukemia (RS4;11), T-acute lymphoblastic leukemia (CCRF-CEM and Jurkat), human promyelocytic cells (HL-60), and human colon adenocarcinoma (HT-29) cells were grown in RPMI medium (Gibco, Milan, Italy). Both media were supplemented with 115 units/mL of penicillin G (Gibco, Milan, Italy), $115 \mu \mathrm{g} / \mathrm{mL}$ of streptomycin (Invitrogen, Milan, Italy) and 10\% FBS (Invitrogen, Milan, Italy). Cell lines were tested for mycoplasma contamination every 6 month by RT-PCR analysis. Stock solutions $(10 \mathrm{mM})$ of the different compounds were obtained by dissolving them in DMSO. Individual wells of 96-well tissue-culture microtiter plates were inoculated with $100 \mu \mathrm{L}$ of complete medium containing $5 \times 10^{3}$ adherent cells or $25 \times 10^{3}$ leukemia cells. The plates were incubated at $37^{\circ} \mathrm{C}$ in a humidified $5 \% \mathrm{CO}_{2}$ incubator for $18 \mathrm{~h}$ prior to the experiments. After medium removal, $100 \mu \mathrm{L}$ of fresh medium containing the test compound at different concentrations was added to each well and incubated at $37^{\circ} \mathrm{C}$ for $72 \mathrm{~h}$. Cell viability was assayed by the (3-(4,5-dimethylthiazol-2-yl)-2,5-diphenyl tetrazolium bromide (MTT) test as previously described [3].The GI50 was defined as the compound concentration required to inhibit cell proliferation by $50 \%$, in comparison with cells treated with the maximum amount of DMSO $(0.25 \%)$ and considered as $100 \%$ viability.

\subsection{Antiproliferative activity in Peripheral Blood lymphocytes (PBL)}

Additional experiments were conducted with peripheral blood lymphocytes (PBL) from healthy donors obtained as described previously [21]. For cytotoxicity evaluations in proliferating PBL cultures, non-adherent cells were resuspended at $5 \times 10^{5}$ cells $/ \mathrm{mL}$ in growth medium containing 2.5 $\mu \mathrm{g} / \mathrm{mL}$ PHA (Irvine Scientific). Different concentrations of the test compounds were added, and viability was determined $72 \mathrm{~h}$ later by the MTT test. For cytotoxicity evaluations in resting PBL cultures, non-adherent cells were resuspended $\left(5 \times 10^{5}\right.$ cells $\left./ \mathrm{mL}\right)$ and treated for $72 \mathrm{~h}$ with the test compounds, as described above.

\subsection{Cell cycle analysis}

Jurkat cells were treated with the test compounds for $24 \mathrm{~h}$. Cells were harvested by centrifugation and fixed with $70 \%$ (v/v) cold ethanol. Cells were lysed with $0.1 \%(\mathrm{v} / \mathrm{v})$ Triton X-100 containing RNase A and stained with PI. A Beckman Coulter Cytomics FC500 instrument and MultiCycle for Windows software from Phoenix Flow Systems were used to analyze the cells. 


\subsection{Measurement of apoptosis by flow cytometry}

Jurkat cells were treated with the test compounds and after different times stained with both PI, to stain DNA, and annexin V-fluorescein isothiocyanate, to stain membrane PS exposed on the cell surface, following the instructions of the manufacturer (Roche Diagnostics) of the Annexin-V-Fluos reagent.

\subsection{Choline uptake assay}

Choline uptake was determined as previously reported [9]. Briefly, HepG2 cells (200,000 cells/well) were incubated for $10 \mathrm{~min}$ at $37^{\circ} \mathrm{C}$ either in a medium containing different concentrations of ChoK $\alpha 1$ inhibitors or only with medium as controls. The medium was then removed and the cells immediately exposed to a medium containing [methyl- ${ }^{14} \mathrm{C}$ ]choline $(16 \mu \mathrm{M}, 31 \mathrm{Ci} / \mathrm{mol})$ for $10 \mathrm{~min}$ at 37 ${ }^{\circ} \mathrm{C}$. The incorporation of choline was stopped by medium aspiration followed by two washes with ice-cold PBS containing $580 \mu \mathrm{M}$ choline. The cells were solubilized in $\mathrm{NaOH} 0.1 \mathrm{~N}$ and an aliquot used to determine the total amount of radiolabel taken up by the cells.

\section{Results and Discussion}

\subsection{Preliminary docking studies}

Human choline kinase $\alpha 1$ (hChoK $\alpha 1$; PDB id: 3G15) was chosen for the docking studies due to several reasons: (i) when 3D structures of both ChoK $\alpha 1$ (PDB ID: 3G15) and ChoK $\beta$ (PDB ID: 3FEG) isoforms were crystalized in complex with the first known inhibitor of ChoK, HC-3, HC-3 suffered a phosphorylation on the morpholinium moiety inserted into the choline binding site of the ChoK $\beta$ isoform, thus the obtained crystal structure of the complex was constituted by ChoK $\beta, A D P$, and phosphohemicolinium-3 (PHC-3). This situation did not occur with $\mathrm{ChoK} \alpha$, where was possible to study the enzyme co-crystallized in complex with both, not phosphorylated HC-3 and ADP, therefore (ii) ChoK $\alpha 1$ co-crystallized with both ADP and HC-3 is more appropriated for docking studies in both binding sites than $C h o K \beta$ and (iii) described biological results indicate that is ChoK $\alpha 1$ and not $\mathrm{ChoK} \beta$ is the most suitable target to study and design new anticancer drugs [8]. We published the crystal structure of the ChoK $\alpha 1$ isoenzyme with two inhibitors characterized by a cationic head; the first one named Compound 5: 1-[4-(4-\{4-[(6-amino-9H-purin-9-yl) methyl] phenyl\} butyl)benzyl]-4-(dimethylamino)-pyridinium (PDB ID: 3ZM9; see Figure 2A) [16-17] and a second one called Compound 1-(4-\{4-[(6-amino-3H-purin-3-yl)methyl]phenyl\}benzyl)-4-(dimethylamino)-pyridinium (PDB ID: 4BR3; see Figure 2B) [17]. The resemblances between compounds 5 and $\mathbf{6}$ with the compounds $\mathbf{3 a}-\mathbf{h}$ and $4 \mathrm{a}-\mathrm{h}$ vindicate the use of these novel crystal structures obtained instead of the one provided with HC-3 to perform docking studies given that these $3 \mathbf{a}-\mathbf{h}$ and $\mathbf{4 a}-\mathbf{h}$ structures are characterized in like manner by one cationic head (Figure 3 and Figure S1 and S2). 

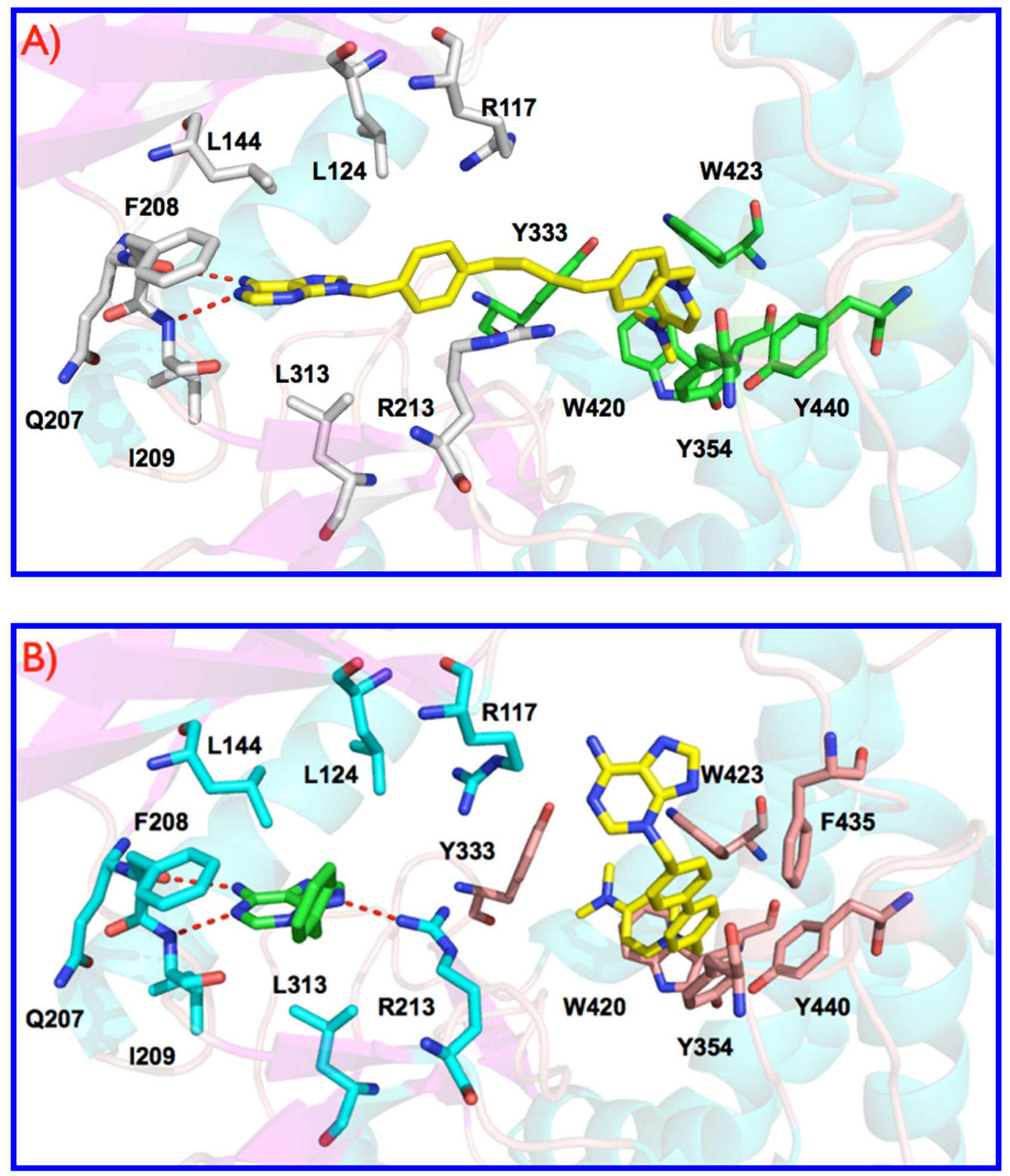

Figure 2. A) Crystal structure of ChoK- $\alpha 1 / 5$ complex (PDB ID: 3ZM9). Compound 5 (carbon atoms in yellow colour) is inserted into ATP (carbon atoms in white colour) and Cho (carbon atoms in green colour) binding sites. B) Crystal structure of ChoK- $\alpha 1 / 6$ complex (PDB ID: 4BR3). One molecule of compounds 6 (carbon atoms in green colours) is inserted into the ATP binding site (carbon atoms in slate blue colour) and another one (carbon atoms in yellow colour) is inserted into the Cho binding site (carbon atoms in salmon colour). Compound 5: 1-[4-(4-\{4-[(6-amino-9H-purin-9-yl)methyl]phenyl\}butyl)benzyl]-4-(dimethylamino)pyridinium bromide Compound 6: 1-(4-\{4-[(6-amino-3H-purin-3-yl)methyl]phenyl\}benzyl)-4-(dimethylamino)pyridinium bromide. 


\subsection{Inhibition of ChoK $\alpha 1$ by compounds $\mathbf{3} \boldsymbol{a}-\boldsymbol{h}$ and $\mathbf{4} \boldsymbol{a}-\boldsymbol{h}$ and docking studies}

Table 1 summarizes the clogP, the inhibitory effect on purified human ChoK $\alpha 1$ activity and the growth inhibitory effects against a panel of six different human tumor-cell lines. Of all the compounds tested, in general terms, those where the aminophenol system is connected by the $\mathrm{N}$-atom (4a-h) are the ones that offer the best results in terms of enzyme inhibition. Among them, compound $4 \mathrm{f}$ stands out with an $\mathrm{IC}_{50}: 0.99 \mu \mathrm{M}$ values very similar to the reference compound MN48b.

As for the length of the spacer, it does not seem to affect the enzyme inhibition; however a significant increase of the inhibitory activity is observed when the spacer is longer in compounds connected by the O-atom the aminophenol group, see compounds (3a-b vs. 3c-d and 3e-f vs. 3g-h). This difference is not apparent when the compounds are by $\mathrm{N}$-atom connected the aminophenol group (4a-h) where the spacer does not seem to exert much influence. On the other hand, the activity is favored by the influence of the substituent in position 4 of the pyridinium ring, where the compounds with dimethylamino (3e-h) and (4e-h) show an appreciable improvement of the inhibition values. As explained in paragraph 3.1, obtained crystal structures of the ChoK $\alpha 1$ isoenzyme with monocationic compounds $\mathbf{5}$ and $\mathbf{6}$ were considered more appropriate for docking studies of compounds $\mathbf{3 a}-\mathbf{h}$ and 4a-h which only have a cationic head than the crystal structure of the enzyme with HC-3.

Figure 2 shows a representation of the interactions of molecules 5 and $\mathbf{6}$ individually with the enzyme. The higher compound (PDB ID: 3ZM9, previously called number 5, see Figure 2A) [16] has an adenine moiety and a 4-(dimethylamino)pyridinium cationic head, connected by a long linker [1,4-diphenylbutane]. This compound occupies a long active site from the ATP to the choline (Cho) binding sites (Figure 2A). The linker is connected to the N-9 adenine atom similarly to the ribose-adenine connection in ATP, and for this reason the adenine moiety can mimic the connexion of the ATP cofactor to ChoK, being inserted into the ATP binding site [16]. The adenine moiety is stabilized by means of hydrophobic interactions with Leu144, Phe208, Ile209 and Leu313; and by two H-bonds with Glu207 and Ile209. The benzyl fragment connected to the adenine moiety is also stabilized by hydrophobic interactions with Arg117, Arg213 and Leu124 side chains. Finally, the 4-(dimethylamino)pyridinium fragment of this compound 5 is inserted into the ATP binding site and stabilized by $\pi$-cations interactions with Tyr333, Tyr354, Trp420, Tyr423 and Trp440.

The compound number 6 (PDB ID: 4BR3, see Figure 2B) also have an adenine moiety and a 4-(dimethylamino)pyridinium fragment, and the linker is smaller being connected to the N-3 adenine atom, and for these reasons one molecule of this compound cannot occupy simultaneously both ATP and Cho binding sites similarly to compound 5 [17]. In the crystal structure (Figure 2B) a partial density corresponding to the 3-benzyl adenine fragment of this compound (carbon atoms in green colours) was detected into the ATP binding site, and another whole molecule (carbon atoms in yellow colours) was observed into the Cho binding site. The adenine moiety inserted into the ATP binding site is also stabilized by hydrophobic interactions with Leu144, Phe208, Ile209 and Leu313; and by H-bonds with Glu207, Ile209, similarly to compound 5. An additional H-bond between the adenine N-9 atom and the Arg213 side chain is also observed, due to the high flexibility of this amino acid. The rest of this compound $\mathbf{6}$ inserted in the ATP binding site were not detected probably due to two reasons: i) The 1-benzyl-4-(dimethylamino)pyridinium fragment is situated outside of the protein, showing a high flexibility and a really poor density and ii) The interaction of compound 6 with this region of the protein is not efficient, as was described recently [11]. Finally, the molecule 6 (carbon atoms in yellow colours) inserted into the Cho binding site interacts with the protein very efficiently, being stabilized by $\pi$-cations interactions with Tyr333, Tyr354, Tyr440, Trp420, Trp423, Trp435 and Phe435. In particular, the biphenyl group shows optimal hydrophobic stacking interactions with Tyr354, and the 4-(dimethylamino)pyridinium moiety interacts trough parallel 
$\pi$-cation interaction with Trp420. This new orientation of this compound 6 inside the Cho binding site is accommodated by a conformational change of Tyr333 that have move back and made an extra space in relation to compound 5. The adenine fragment of this molecule inserted into the Cho binding site is outside of the enzyme and does not show interaction with the protein (see Supplementary Figure S1B), being the 1-(biphenyl-4-ylmethyl)-4-(dimethylamino)pyridinium the key fragment of this compound for the interaction into the ATP binding site [17].

Docking studies have been performed in both crystal structures and the analysis of the obtained poses indicates which compounds could be similar to compound 5 or to compound $\mathbf{6}$. In fact, compounds $\mathbf{3 a}, \mathbf{3 b}, \mathbf{3 e}, \mathbf{3 f}, \mathbf{4 a}, \mathbf{4 b}, \mathbf{4 e}$ and $\mathbf{4 f}$ (Figure 3 and Figure $\mathbf{S 1}$ of the Supporting Information) have shown good poses in the crystal structure of compound $\mathbf{6}$, and the correct poses of compounds $3 \mathrm{c}, 3 \mathrm{~d}, 3 \mathrm{~g}, 3 \mathrm{~h}, 4 \mathrm{c}, 4 \mathrm{~d}, 4 \mathrm{~g}$ and $4 \mathrm{~h}$ (Figure 3 and Figure S2 of the Supporting Information) were obtained in the crystal structure of compound 5 . The reason for these poses is the length of the linker. In fact, all compounds that have a benzene or biphenyl linker are inserted into the ChoK binding site similarly to compound $\mathbf{6}$, and compounds that have a 1,2-diphenylethane or a 1,4-diphenylbutane linker are inserted into the ATP and ChoK binding sites, similarly to compound 5.

Table 1. In vitro inhibitory effects of compounds 3a-h and 4a-h.

\begin{tabular}{|c|c|c|c|c|c|c|c|c|}
\hline \multirow[t]{2}{*}{ Comp. } & \multirow[t]{2}{*}{$\operatorname{cog} P^{a}$} & \multirow{2}{*}{$\begin{array}{c}\mathrm{IC}_{50^{\mathrm{b}}}(\mu \mathrm{M}) \\
h \mathrm{ChoK} \alpha 1\end{array}$} & \multicolumn{6}{|c|}{$\mathbf{G I}_{50^{c}}(\mu \mathrm{M})$} \\
\hline & & & HT-29 & HeLa & MCF-7 & CCRF-CEM & HL-60 & RS4;11 \\
\hline $3 a$ & 0.68 & $>50$ & $36.0 \pm 7.4$ & $4.8 \pm 0.58$ & $22.8 \pm 4.3$ & $3.4 \pm 0.7$ & $4.8 \pm 0.4$ & $16.8 \pm 4.8$ \\
\hline $3 b$ & 2.57 & $17.55 \pm 1.24$ & nd & nd & nd & nd & nd & nd \\
\hline $3 c$ & 3.13 & $8.56 \pm 0.94$ & $0.63 \pm 0.14$ & $0.068 \pm 0.028$ & $0.3 \pm 0.1$ & $0.03 \pm 0.003$ & $0.011 \pm 0.005$ & $0.15 \pm 0.012$ \\
\hline $3 d$ & 4.18 & $6.79 \pm 0.47$ & $0.58 \pm 0.17$ & $0.16 \pm 0.048$ & $0.5 \pm 0.2$ & $0.015 \pm 0.005$ & $0.12 \pm 0.091$ & $0.29 \pm 0.053$ \\
\hline $3 e$ & 0.57 & $6.33 \pm 0.80$ & $26.1 \pm 6.7$ & $8.2 \pm 2.4$ & $23.6 \pm 5.1$ & $1.2 \pm 0.5$ & $38.7 \pm 6.2$ & $53.6 \pm 13.4$ \\
\hline $3 f$ & 2.45 & $6.39 \pm 0.46$ & $4.4 \pm 0.7$ & $4.2 \pm 0.5$ & $7.6 \pm 1.6$ & $0.54 \pm 0.21$ & $2.4 \pm 0.7$ & $2.0 \pm 0.5$ \\
\hline $3 g$ & 3.01 & $4.29 \pm 0.12$ & $2.2 \pm 0.9$ & $1.2 \pm 0.2$ & $4.3 \pm 0.9$ & $0.40 \pm 0.26$ & $1.6 \pm 0.9$ & $2.6 \pm 0.2$ \\
\hline $3 \mathrm{~h}$ & 4.07 & $7.01 \pm 0.13$ & nd & nd & nd & nd & nd & nd \\
\hline $4 a$ & 0.33 & $3.34 \pm 0.34$ & $49.7 \pm 11.4$ & $12.8 \pm 2.6$ & $17.8 \pm 5.3$ & $7.3 \pm 2.5$ & $4.1 \pm 1.2$ & $24.5 \pm 2.9$ \\
\hline $4 b$ & 2.22 & $3.85 \pm 0.10$ & $6.6 \pm 2.3$ & $2.1 \pm 0.4$ & $4.4 \pm 0.5$ & $0.5 \pm 0.1$ & $1.1 \pm 0.3$ & $2.8 \pm 0.6$ \\
\hline $4 c$ & 2.78 & $3.83 \pm 0.91$ & $5.3 \pm 2.1$ & $0.86 \pm 0.26$ & $1.7 \pm 0.4$ & $0.14 \pm 0.03$ & $0.065 \pm 0.023$ & $0.46 \pm 0.061$ \\
\hline $4 d$ & 3.84 & $3.36 \pm 0.17$ & $5.8 \pm 1.9$ & $0.39 \pm 0.12$ & $2.8 \pm 0.5$ & $0.086 \pm 0.03$ & $0.33 \pm 0.074$ & $0.71 \pm 0.16$ \\
\hline $4 e$ & 0.22 & $2.82 \pm 0.16$ & $23.8 \pm 8.2$ & $12.42 \pm 1.8$ & $6.7 \pm 1.5$ & $8.4 \pm 1.6$ & $4.0 \pm 0.9$ & $31.0 \pm 2.6$ \\
\hline $4 f$ & 2.10 & $0,99 \pm 0,17$ & $13.2 \pm 5.0$ & $3.6 \pm 0.4$ & $5.0 \pm 1.0$ & $1.1 \pm 0.4$ & $1.3 \pm 0.4$ & $3.7 \pm 0.4$ \\
\hline $4 g$ & 2.66 & $3.66 \pm 0.11$ & $5.2 \pm 1.7$ & $1.7 \pm 0.2$ & $2.4 \pm 0.2$ & $0.7 \pm 0.1$ & $0.25 \pm 0.066$ & $1.1 \pm 0.3$ \\
\hline $4 h$ & 3.72 & $6.39 \pm 0.46$ & $3.6 \pm 1.4$ & $0.7 \pm 0.1$ & $2.2 \pm 1.0$ & $0.16 \pm 0.034$ & $0.37 \pm 0.079$ & $0.5 \pm 0.08$ \\
\hline$M N-48 b^{d}$ & 1.27 & $0.78 \pm 0.03$ & $1.9 \pm 0.4$ & $1.9 \pm 0.1$ & $1.8 \pm 0.06$ & $0.21 \pm 0.09$ & $0.32 \pm 0.03$ & $0.17 \pm 0.04$ \\
\hline RSM-932Ad & 4.13 & $1.92 \pm 0.06$ & $0.4 \pm 0.2$ & $0.83 \pm 0.1$ & $0.18 \pm 0.10$ & $0.61 \pm 0.20$ & $0.93 \pm 0.1$ & $1.0 \pm 0.3$ \\
\hline $\begin{array}{l}{ }^{\mathrm{a} T h e} \\
{ }^{\mathrm{b}} \mathrm{IC}_{50} \\
{ }^{\mathrm{c}} \mathrm{GI}_{50} \\
{ }^{\mathrm{d} D a t} \text { Da } \\
\mathrm{nd}=\mathrm{n}\end{array}$ & $\begin{array}{l}\mathrm{s} \text { of clogl } \\
\text { npound } \\
\text { npound } \\
\mathrm{n} \text { from } \mathrm{r}\end{array}$ & $\begin{array}{l}\text { re calculated } \\
\text { entration requ } \\
\text { entration requ } \\
\text { 9]. }\end{array}$ & $\begin{array}{l}\text { ith Chemdr } \\
\text { red to inhib } \\
\text { red to inhib }\end{array}$ & $\begin{array}{l}\text { v } 15.0 \text {. } \\
\text { ChoK } \alpha 1 \text { enz } \\
\text { tumor cell pr }\end{array}$ & $\begin{array}{l}\text { he by } 50 \% \text {. } \\
\text { iferation by }\end{array}$ & & & \\
\hline
\end{tabular}


As an example, Figure 3 (Panel A and B) shows the obtained poses of compounds $3 \mathbf{f}$ (carbon atoms in white colour) and $4 \mathrm{f}$ (carbon atoms in orange colour). Both compounds have a 4-(dimethylamino)pyridinium cationic head and a biphenyl linker. The cationic head in these compounds is also stabilized by $\pi$-cation interaction and the biphenyl group shows optimal hydrophobic stacking interactions, being very similar to compound $\mathbf{6}$. Nevertheless, in compound $3 \mathrm{f}$ the linker is connected to the 3-aminophenol $\mathrm{O}$-atom, and in compound $4 \mathrm{f}$ the linker is connected to the 3-aminophenol $\mathrm{N}$-atom. The 3-aminophenol fragment of compound $4 \mathrm{f}$ is stabilized by two H-bonds, with Tyr354 and Glu434, while in compound $3 \mathrm{f}$ is stabilized by only one H-bond with Tyr354, and for this reason compound $4 \mathbf{f}$ has lower IC $50(0,99 \pm 0,17 \mu \mathrm{M})$ compared to $3 \mathbf{f}(6,39 \pm 0,46$ $\mu \mathrm{M})$ regarding the inhibition of ChoK $\alpha 1$. Concerning compounds $\mathbf{3} \mathbf{a}, \mathbf{3} \mathbf{b}, \mathbf{3 e}, \mathbf{4} \mathbf{a}, \mathbf{4} \mathbf{b}$, and $4 \mathbf{e}$, the obtained poses highlights that the increased size of the 4-(pyrrolidin-1-yl)pyridininium cationic head ( $\mathbf{a} \mathbf{a}$ and $\mathbf{3} \mathbf{b}$ ) negatively affects the $\pi$-cations interactions (Figure S1), resulting in a larger IC 50 compared to the equivalents compounds with a 4-(dimethylamino)pyridinium (3e). On the other hand, the biphenyl linker connected to the 3-aminophenol $\mathrm{N}$-atom (compound $\mathbf{4 b}$ ) allows two H-bonds with Tyr354 and Glu434 and hence increases the inhibitory activity. Figure 3 also shows the obtained pose of compounds $3 \mathrm{~g}$ (carbon atoms in cyan colours) and $\mathbf{4 g}$ (carbon atoms in magenta colours), which also confirm the previous hypotheses. With respect to the $3 \mathbf{h}$ compounds, it does not seem to affect in this case the volume of the substituent in position 4 of the cationic head in the case of the family containing the -O-atom of the aminophenol connected to rest of the molecule, while in their isomers ( $4 \mathbf{d} v s .4 \mathbf{h})$ an unexplained decrease of the enzymatic activity of $4 \mathbf{h}$ is observed (Figure S2)

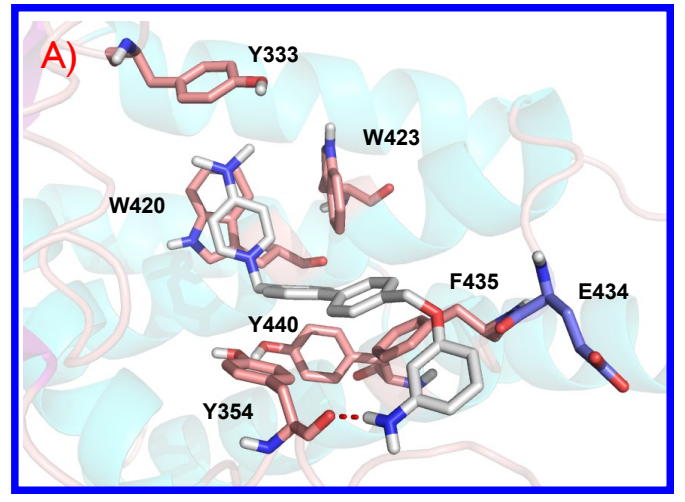

Compound $3 f$

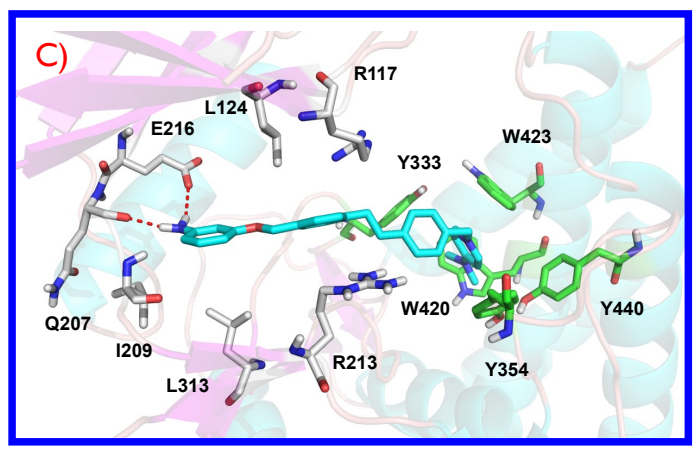

Compound $3 g$

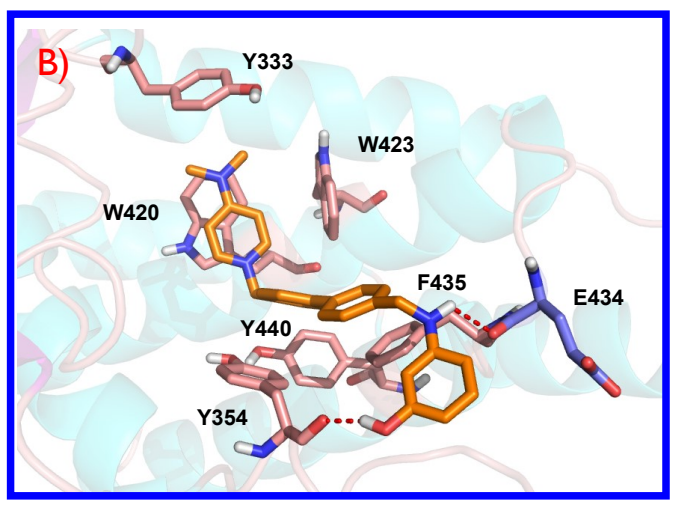

Compound $4 \mathbf{f}$

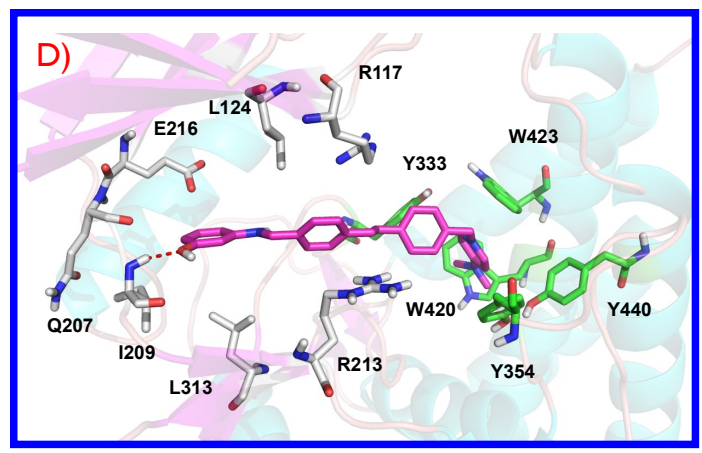

Compound $\mathbf{4 g}$

Figure 3. Obtained pose of compounds $3 \mathrm{f}(\mathrm{A}$, carbon atoms in white colours) and $\mathbf{4 f}(\mathrm{B}$, carbon atoms in orange colours) inserted into de Cho binding site similarly to compound 6 (PDB ID: 4BR3), and compounds 3g (C, carbon atoms in cyan colours) and $\mathbf{4 g}$ (D, carbon atoms in magenta colours) inserted in both ATP and Cho binding sites similarly to compound 5 (D, PDB ID: 3ZM9) 


\subsection{In vitro antiproliferative activities}

The derivative $3 \mathrm{c}$ was the most active compound identified in this study, inhibiting the growth of HT-29, HeLa, MCF-7, CCRF-CEM, HL-60 and RS4;11cells with GI50 values ranging from 630 to 11 $\mathrm{nM}$, resulting 30 -fold more potent than $\mathrm{MN}-48 \mathrm{~b}$ against HL-60 leukemic cells.

With respect to the effect of the rest of final compounds, it can be observed that: the compounds that offer inhibition values at the nanomolar values correspond to $3 \mathbf{c}-\mathbf{d}$ and $4 \mathbf{c}-\mathbf{d}$, i.e. those with the longest spacer ( 2 or 4 carbons). In addition, these compounds are also those that possess a pyrrolidine as a constituent in position 4 of the pyridinium ring. There are no major differences in activity between the compounds linked by oxygen and those linked by nitrogen, although the most active compound corresponds to $3 \mathrm{c}$. The fact that the compounds with the best IC $\mathrm{C}_{50}$ values of enzyme inhibition do not correspond to those with the best antiproliferative values may be due to differences in $\operatorname{cog} \mathrm{P}$, which in turn lead to an increase in membrane uptake. Indeed, $\mathbf{3 c - d}$ and $\mathbf{4 c - d}$ have higher $\operatorname{cog} \mathrm{P}$ values than their counterparts with shorter spacers (3a-b and $\mathbf{4} \mathbf{a}-\mathbf{b})$. On the other hand, compounds with dimethylamino substituents on the 4 of the pyridinium ring show a significant reduction in enzyme inhibition, which could be due to the larger volume of the 4-(pyrrolidin-1-yl)pyridinium cationic head produces a lower $\pi$-cation interaction, and these molecules show a lower $\mathrm{IC}_{50}$ of the enzyme relative to the equivalent compounds with a 4-(dimethylamino)pyridinium mentioned above.

\subsection{Effects of compound $3 \mathrm{c}$ and $4 \mathrm{f}$ in non-tumor cells.}

To investigate the cytotoxic potential of these compounds in normal human cells, the two compounds $3 \mathbf{c}$ and $\mathbf{4 f}$ were evaluated in vitro against peripheral blood lymphocytes (PBL) collected from healthy donors. Compound $3 \mathrm{c}$ showed an GI50 greater than $10 \mu \mathrm{M}$, both in quiescent lymphocytes and in proliferating lymphocytes stimulated with phytohemagglutinin (PHA) (Table 2), suggesting that this compound specifically targets tumoral cells. On the other hand compound $4 \mathrm{f}$ exhibits a minimal toxicity with GI50 of $7.6 \mu \mathrm{M}$ and $3.6 \mu \mathrm{M}$ in quiescent and PHA-stimulated lymphocytes respectively. Nevertheless these values were almost 120 times higher than that observed against the T-lymphoblastic cell line (CCRF-CEM).

Table 2. Cytotoxicity of compounds $3 \mathbf{c}$ and $\mathbf{4 f}$, for human peripheral blood lymphocytes (PBL)

\begin{tabular}{lcc}
\hline & \multicolumn{2}{c}{$\mathrm{GI}_{50}(\mu \mathrm{M})^{\text {a }}$} \\
\cline { 2 - 3 } & $3 \mathbf{c}$ & $\mathbf{4 f}$ \\
PBLresting $^{\mathrm{b}}$ & $>10$ & $7.6 \pm 1.1$ \\
PBLPHA $^{\mathrm{c}}$ & $>10$ & $3.6 \pm 0.8$
\end{tabular}

${ }^{a}$ Compound concentration required to inhibit cell growth by 50\%. ${ }^{b}$ PBL not stimulated with PHA.

c PBL stimulated with PHA.Values are the mean \pm SEM for three separate experiments.

\subsection{Cell cycle analysis}

To study in detail the mechanism of action of these compounds we used a T-acute lymphoblatic leukemia cell line (Jurkat) against which we demonstrated a particular efficacy of the ChoK $\alpha 1$ inhibitors [12]. We first studied the effects on cell cycle following treatment with the two compounds and the results are shown in Figure 3. As can be seen, both compounds cause only modest and not significative changes in the cell cycle even at the highest concentration used $(5 \mu \mathrm{M})$ 
A

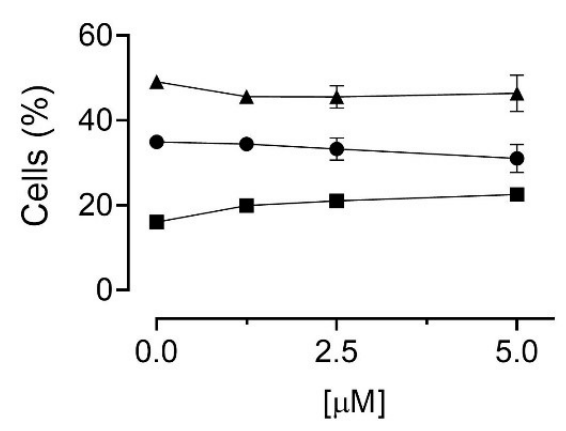

C

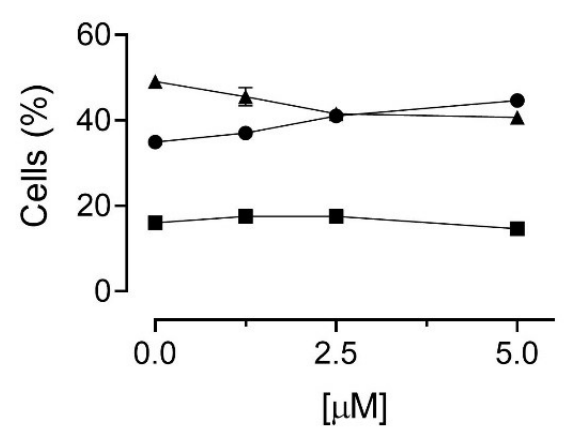

B

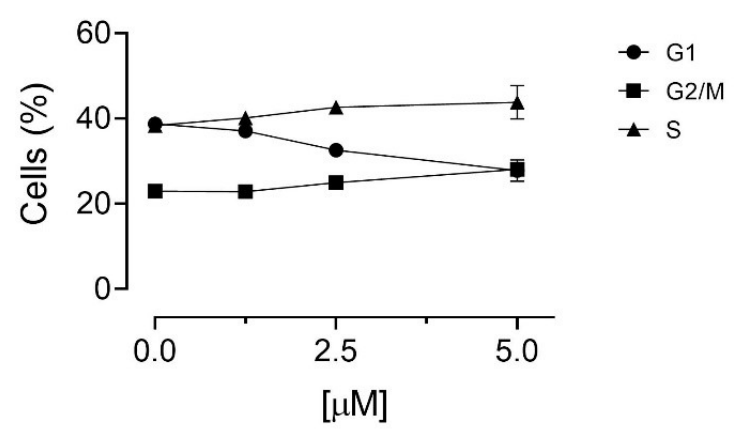

$\mathrm{D}$

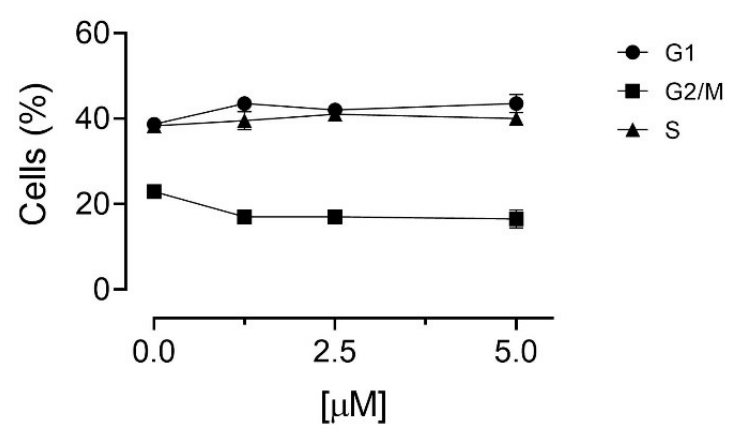

Figure 3. Effect of $4 \mathbf{f}$ (panels $A$ and B) and 3c (panels $C$ and D) on cell cycle in Jurkat cells. Cells were treated with the compounds for $24 \mathrm{~h}$ (panels A and C) and $48 \mathrm{~h}$ (panels B and D), at the concentrations of 1.25, 2.5, and $5.0 \mu \mathrm{M}$. Cells were then fixed and labeled with PI and analyzed by flow cytometry as described in the experimental section. Data are represented as mean of two separate experiments \pm SEM.

\subsection{Measurement of apoptosis by flow cytometry}

To better depict the cytotoxic effect of compounds $\mathbf{3 c}$ and $\mathbf{4 f}$, Jurkat cells were subjected to double labeling with annexin-V and PI and then analysed by flow cytometry. This procedure allows the quantitation of four different cell populations: living cells (annexin- $-/ / \mathrm{PI}-$ ), early apoptotic cells (annexin- $\mathrm{V}^{+} / \mathrm{PI}$ ), late apoptotic cells (annexin- $\mathrm{V}^{+} / \mathrm{PI}^{+}$) and necrotic cells (annexin- $-\mathrm{V}^{-} / \mathrm{PI}^{+}$). Jurkat cells treated with 3c and 4f (Figure 4) displayed a significant increase of apoptotic cells afte $24 \mathrm{~h}$ of treatment in a concentration dependent manner. 

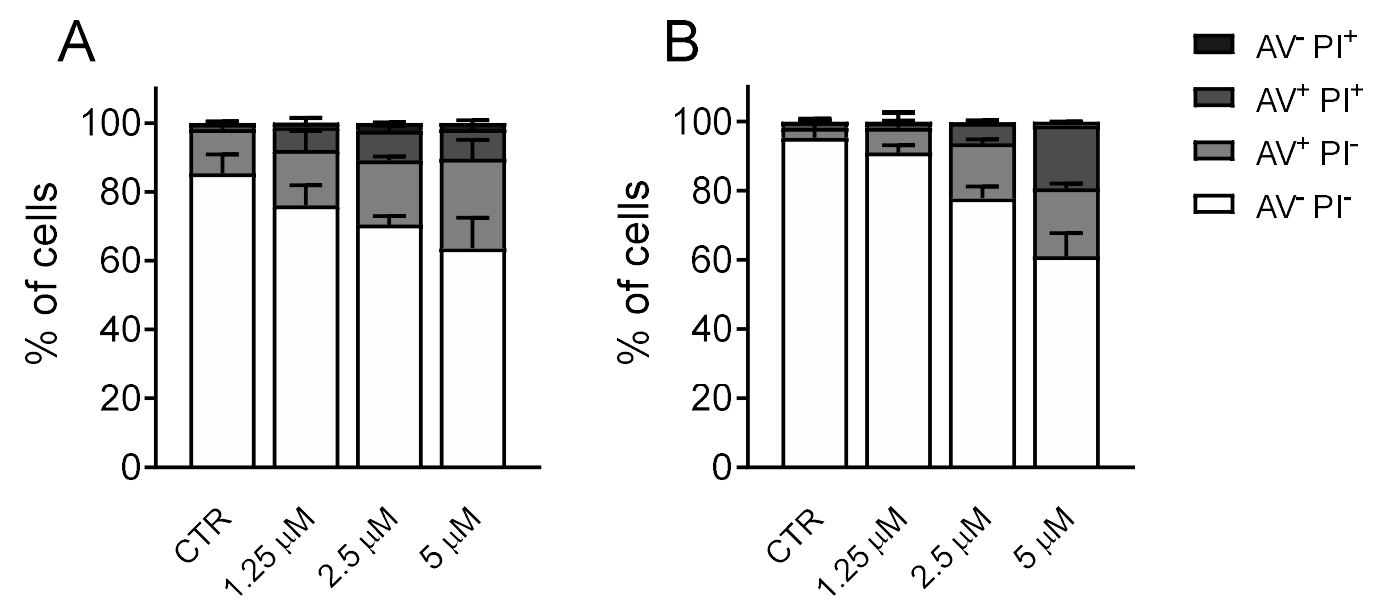

Figure 4. Flow cytometric analysis of apoptotic cells after treatment of Jurkat cells with compounds $4 \mathrm{f}$ (panel A) and $3 \mathbf{c}$, (panel B) at the indicated concentrations after incubation for $48 \mathrm{~h}$. The cells were then harvested and labeled with annexin-V-FITC and PI and analyzed by flow cytometry. Data are represented as mean \pm SEM of three independent experiments.

\subsection{Choline uptake assay}

Since recently we has been previously demonstrated that some ChoK $\alpha 1$ inhibitors, in addition to their effects on $h \mathrm{ChoK} \alpha 1$ activity, are able to reduce choline uptake into the cell [9], we decided to investigate whether these compounds were also able of inhibiting choline uptake in HepG2 cells. The two compounds chosen were $\mathbf{3 d}$ (Table 1 and Table 2); which shows moderate enzyme inhibition but very good antiproliferative values. On the other hand, the other compound chosen, $4 \mathbf{f}$, has a good inhibitory IC 50 but modest GI50 values on cell growth (Table 1 and Table 2). Compound 3d inhibits choline uptake moderately, so its high antiproliferative activity cannot be directly attributed only to choline uptake but to a dual effect between choline uptake and enzyme inhibition. In contrast, the compound with poor effect on cell growth inhibition $\mathbf{4 f}$, has good enzyme and choline uptake inhibition values, 4 f shows low lipophilicity, which perhaps does not allow the compound to pass through the plasma membrane. Therefore, it follows that a good value for choline uptake and enzyme inhibition is not sufficient, and the need to control the lipophilicity of inhibitors to allow the molecules to pass through the plasma membrane comes into play in the equation.

Table 2. IC $50(\mu \mathrm{M})$ Inhibition of $[3 \mathrm{H}]$ choline uptake by compounds $\mathbf{3 d}$ and $\mathbf{4 f}$ in HepG2 cells.

\begin{tabular}{c|c} 
Compounds & IC $_{50}($ choline uptake, $\mu \mathrm{M})$ \\
\hline $\mathbf{3 d}$ & $15.8 \pm 0.24$ \\
$\mathbf{4 f}$ & $3.5 \pm 0.07$
\end{tabular}




\section{Conclusions}

The initial hypothesis suggested that the derivative compounds analyzed in this work with a longer linker would potent hChoK $\alpha 1$ inhibitors, since they could be inserted in both ATP and Cho binding sites of the hChoK $\alpha 1$ enzyme simultaneously. According to this premise, compounds $\mathbf{3 c}, \mathbf{3 d}, \mathbf{3 g}, \mathbf{3 h}$, $4 \mathrm{c}, 4 \mathrm{~d}, 4 \mathrm{~g}$ and $4 \mathrm{~h}$ whose results obtained after docking studies and poses analyzing indicated that they could be similar to model compound so-called number 5 (PDB ID: 3ZM9) should be the more potent. However the compound $\mathbf{4 f}$ (a compound that shown good poses in the crystal structure of compound 6 (PDB ID: 4BR3), the one with smaller linker) shows the better IC ${ }_{50}(0.99 \pm 0.17 \mu \mathrm{M})$ for the inhibition of ChoK $\alpha 1$. This can be explained because not one molecule occupying the ATP and Cho binding sites all at once was found but two wholes molecules, one in the ATP binding site and another into the Cho binding site were detected. The reason which explained a higher $\mathrm{IC}_{50}$ for its counterpart $3 \mathrm{f}(6.39 \pm 0.46 \mu \mathrm{M})$ would be that the linker is connected to the 3-aminophenol O-atom and this moiety is stabilized by only one H-bond with Tyr354 while the 3-aminophenol fragment of compound $4 \mathrm{f}$ is stabilized by two H-bonds, with Tyr354 and Glu434 provided by both hydrogens from $-\mathrm{NH}$ - and $\mathrm{OH}$ groups (Figure 3; $3 \mathbf{A}$ and $3 \mathbf{B}$ ). Nevertheless antiproliferative effects must be explained taking into account not only the inhibition of the enzyme but also the clogP, a measure of lipophilicity of a compound. In accordance with these two parameters, the better antiproliferative activities are obtained with compounds endowed with high $\operatorname{cog} \mathrm{P}(\mathbf{3 c}, \mathbf{3 d}, \mathbf{4 c}, 4 \mathrm{~d})$, a consequence of a longer linker and two additional carbons in the pyrrolidine fragment. Interestingly these compounds appear to have low toxicity as they have no significant effect on either quiescent or PHA-stimulated human lymphocytes. They also induce apoptosis in a dose dependent manner in Jurkat cells. Choline uptake assays also highlight the dual target of these compounds, where lipophilicity plays an essential role in the antitumour capacity of the compounds without ruling out that other mechanisms may contribute to antiproliferative SAR.

Author Contributions: Conceptualization and methodology LC.L-C, M.P. C-J and G.V., synthesis and doking studies S.S-O, P.M.L-N writing — original draft preparation L.C. L-C. M-K-S and G.V. Purification of ChoK $\alpha 1$ R. H-G. Biological Assays M.P. C-J, P.R.M. and E.M., supervision, LC.L-C, M.P. C-J and G.V.; funding acquisition, L.C.L-C.; All authors have read and agreed to the published version of the manuscript.

Funding: "This research was funded by Convocatoria 2019 Proyectos de I + D + i - RTI Tipo B “Ministerio de Innovación y Ciencia” grant number PID2019-109294RB-I00 and University of Granada, Cei-BioticProject grant number CEI2013-MP-1.

Conflicts of Interest: The authors declare no conflict of interest.

\section{References}

[1] Gibellini, F.; Smith, T.K. The Kennedy Pathway-De Novo Synthesis of Phosphatidylethanolamine and Phosphatidylcholine. IUBMB Life. 2010, 62, 414.

[2] Kanno, K.; Wu, M. K.; Scapa, E.F.; Roderick, S.L.; Cohen, D.E. Structure and Function of Phosphatidylcholine Transfer Protein (PC-TP)/StarD2. Biochim Biophys Acta, 2007, 1771, 654.

[3] Castro-Navas, F.F.; Schiaffino-Ortega, S.; Carrasco-Jimenez, M.P.; RiosMarco, P.; Marco, C.; Espinosa, A.; Gallo, M.A.; Mariotto, E.; Basso, G.; Viola, G.; Entrena-Guadix, A.; Lopez-Cara, L.C. New more polar symmetrical bipyridinic compounds: new strategy for the inhibition of choline kinase alpha 1. Future Med. Chem. 2015, 7, 417.

[4] Estévez-Braun, A.; Ravelo, A.G.; Pérez-Sacau, E.; Lacal J.C. A new family of choline kinase inhibitors with antiproliferative and antitumor activity derived from natural products. Clin Transl Oncol. 2015,17, 74.

[5] Rodríguez-González, A.; Ramírez de Molina A, Fernández, F.; Lacal J.C. Choline kinase inhibition induces the increase in ceramides resulting in a highly specific and selective cytotoxic antitumoral strategy as a potential mechanism of action. Oncogene. 2004, 23, 8247.

[6] Gokhale, S.; Xie, P. ChoK-Full of Potential: Choline Kinase in B Cell and T Cell Malignancies. Pharmaceutics. 2021, 13, 911. 
[7] Lacal, J.C.; ZimmermanT.; Campos, J.M. Choline Kinase: An Unexpected Journey for a Precision Medicine Strategy in Human Diseases. Pharmaceutics. 2021, 13, 788.

[8] Rubio-Ruiz, B.; Serran-Aguilera, L.; Hurtado-Guerrero, R.; Conejo-Garcia, A. Recent advances in the design of choline kinase alpha inhibitors and the molecular basis of their inhibition. Med. Res. Rev. 2021, 41, 902927.

[9] Serrán Aguilera,L.; Mariotto, E.; Rubbini, G.; Franci Castro Navas, F.F.; Marco, C.; Carrasco-Jiménez, M. P.; Ballarotto, M.; Macchiarulo, A.; Hurtado-Guerrero, R.; Viola, G.; Lopez-Cara, L.C. Synthesis, Biological evaluation, in silico modeling and Crystallisation of novel small monocationic molecules with potent antiproliferative activity by dual mechanism. Eur. J. Med. Chem. 2020, 207, 112797.

[10] Sola-Leyva, A.; López-Cara, L.C.; Ríos-Marco, P.; Ríos, A.; Marco, C. Carrasco-Jimenez M. P.; Choline kinase inhibitors EB-3D and EB-3P interferes with lipid homeostasis in HepG2 cells. Sci. Rep.2019, 9, 5109.

[11] Jabalera,Y.; Sola-Leyva, A.; Peigneux, A.; Vurro, F.; Iglesias, G.R.; Vilchez-Garcia, J.; Inmaculada Pérez-Prieto,I.; Aguilar-Troyano,F.J.; López-Cara, L.C.; Carrasco-Jiménez, M.P.; Jimenez-Lopez, C. Biomimetic Magnetic Nanocarriers Drive Choline Kinase Alpha Inhibitor inside Cancer Cells for Combined Chemo-Hyperthermia Therapy. Pharmaceutics. 2019, 12, 8.

[12] Mariotto, E.; Bortolozzi, R.; Volpin, I.; Carta, D.; Serafin, V.; Accordi, B.; Basso, G.; Luque Navarro, P.; López-Cara, L.C.; Viola, G. EB-3D a novel choline kinase inhibitor induces deregulation of the AMPK-mTOR Pathway and apoptosis in leukemia T-cells. Biochem. Pharmacol. 2018. 155, 213.

[13] Mariotto, E.; Viola, G.; Ronca, R.; Persano, L.; Aveic, S.; Bhujwalla, Z.M.; Mori, N.; Accordi, B.; Serafin, V.; López-Cara, L.C.; Bortolozzi, R. Choline Kinase Alpha Inhibition by EB-3D Triggers Cellular Senescence, Reduces Tumor Growth and Metastatic Dissemination in Breast Cancer. Cancers, 2018, 10, 391.

[14] Janardhan, S.; Srivani, P.; Sastry, G.N. Choline Kinase: An Important Target for Cancer. Curr. Med. Chem. 2006, 13, 1169.

[15] Schiaffino-Ortega, S; Lopez-Cara, LC; Rios-Marco, P; Carrasco-Jimenez, MP; Gallo, MA; Espinosa, A; Marco, C; Entrena, A. New non-symmetrical choline kinase inhibitors. Bioorg. Med. Chem. 2013, 21, 7146.

[16] Sahun-Roncero, M; Rubio-Ruiz,, B; Saladino, G; Conejo-Garcia, A; Espinosa, A; Velazquez-Campoy, A; Gervasio, FL; Entrena, A; Hurtado-Guerrero, R. The Mechanism of Allosteric Coupling in Choline Kinase1 Revealed by the Action of a Rationally Designed Inhibitor. Angew. Chem. Int. Ed. 2013, 52, 4582.

[17] Sahún-Roncero, M; Rubio-Ruiz, B; Conejo-Garcia, A; Velázquez-Campoy A; Entrena, A; Hurtado-Guerrero, R. Determination of Potential Scaffolds for Human Choline Kinase $\alpha 1$ by Chemical Deconvolution Studies. ChemBioChem. 2013, 14, 1291.

[18] Rubio-Ruiz, B; Conejo-Garcia, A; Rios-Marco, P; Carrasco-Jimenez, MP; Segovia, J; Marco, C; Gallo, MA; Espinosa, A; Entrena, A. Design, synthesis, theoretical calculations and biological evaluation of new non-symmetrical choline kinase inhibitors. Eur. J. Med. Chem. 2012, 20, 154.

[19] Schiaffino-Ortega, S.; Baglioni, E.; Mariotto, E.; Bortolozzi, R.; Serrán-Aguilera, L.; Ríos-Marco, P.; Carrasco-Jimenez, M.P.; Gallo, M.A.; Hurtado-Guerrero, R.; Marco, C.; et al. Design, synthesis, crystallization and biological evaluation of new symmetrical biscationic compounds as selective inhibitors of human Choline Kinase $\alpha 1$ (ChoK_1). Sci. Rep. 2016, 6, 23793.

[20] Rubbini, G.; Buades-Martín, A.B.; Kimatrai-Salvador, M.; Entrena, A.; Gallo-Mezo, M.Á.; Ríos-Marco, P.; Marco, C.; Mattiuzzo, E.; Bortolozzi, R.; Mariotto, E.; et al. Lead optimization-hit expansion of new asymmetrical pyridinium/quinolinium compounds as choline kinase $\alpha 1$ inhibitors. Future Med. Chem. 2018, 10, 1769.

[21] Romagnoli, R.; Baraldi, P.G., Kimatrai Salvador, M.; Schiaffino Ortega, S.; Prencipe, F.; Brancale, A.; Hamel, E.; Castagliuolo, I.; Mitola, S.; Ronca, R.; et al. Design, Synthesis, in Vitro and in Vivo Anticancer and Antiangiogenic Activity of Novel 3-Arylamino Benzofuran Derivatives Targeting the Colchicine Site on Tubulin J. Med Chem. 2015, 58, 3209-3222. 